\title{
Intrahepatic Cholestasis of Pregnancy in Women With Twin Pregnancy
}

\author{
Dan Shan, ${ }^{1}$ Yayi Hu, ${ }^{1}$ Peiyuan Qiu, ${ }^{2}$ Bechu Shelley Mathew, ${ }^{3}$ Yun Chen, ${ }^{1}$ Si Li, ${ }^{1}$ Yuan Hu, Lijun Lin, ${ }^{1}$ \\ Zhi Wang, ${ }^{1}$ and Luping $\mathrm{Li}^{1}$ \\ ${ }^{1}$ Department of Obstetrics and Gynecology, West China Second University Hospital, Sichuan University, Chengdu, \\ Sichuan, China; Key Laboratory of Birth Defects and Related Diseases of Women and Children, Sichuan University, Ministry \\ of Education \\ ${ }^{2}$ West China School of Public Health, Sichuan University, Chengdu, Sichuan, China \\ ${ }^{3}$ Department of Anesthesia, West China Hospital, Sichuan University, Chengdu, Sichuan, China
}

\begin{abstract}
The aim of the present work was to determine maternal and fetal outcomes of intrahepatic cholestasis of pregnancy (ICP) in twin pregnancies. All twin pregnancies delivered above 28 gestational weeks in West China Second University Hospital from January 2013 to May 2015 were included. Data on maternal demographics and obstetric complications together with fetal outcomes were collected. The risk of adverse maternal and fetal outcomes were determined in relation to ICP by crude odds ratios (OR) and adjusted ORs (aOR) with 95\% confidence intervals (Cl). Subgroup analysis concentrated on the effect of assisted reproductive technology (ART), ICP severity, and onset time. A total of 1,472 twin pregnancies were included, of which 362 were cholestasis patients and 677 were conceived by ART. Higher rates of preeclampsia (aOR $1.96 ; 95 \% \mathrm{Cl} 1.35,2.85)$, meconium-stained amniotic fluid (aOR 3.10; 95\% Cl 2.10, 4.61), and preterm deliveries (aOR 3.20; 95\% Cl 2.35, 4.37) were observed in ICP patients. Subgroup analysis revealed higher incidences of adverse outcomes in severe and early onset ICP groups. In conclusion, adverse maternal and fetal outcomes were strongly associated with ICP in twin patients. Active management and close antenatal monitoring are needed, especially in the early onset and severe groups.
\end{abstract}

Keywords: pregnancy, twin, intrahepatic cholestasis of pregnancy, assisted reproductive technologies

Intrahepatic cholestasis of pregnancy (ICP) is a unique, pregnancy-related hepatic disorder characterized by pruritus with disturbed liver function tests and typically elevated serum levels of total bile acids (TBA; Williamson \& Geenes, 2014). The incidence of ICP varies around the globe, geographically between $0.1 \%$ and $15.6 \%$ (Ozkan et al., 2015; Williamson \& Geenes, 2014), and in China, the incidence is about $1.0-4.0 \%$ along the Yangtze River (Li et al., 2013). ICP is associated with increased risk for many fetal complications, such as sudden intrauterine fetal demise and stillbirth (Brouwers et al., 2015; Puljic et al., 2015; Williamson \& Geenes, 2014). The etiology of ICP is not fully understood; however, the contributing factors appear to be multifactorial, including ethnicity, diet, advanced maternal age, family history of biliary disease, ICP in previous pregnancy, and twin gestation (Diken et al., 2014; Williamson \& Geenes, 2014).

In comparison with singleton pregnancies, maternal complications, and perinatal sequelae are more frequent in multiple gestations. Multiple pregnancies tend to increase the risk of maternal mortality, morbidity, and pregnancy- associated complications, such as gestational diabetes mellitus (GDM), gestational hypertensive disorders, and postpartum hemorrhage (Obiechina et al., 2011; Rao et al., 2004; Vogel et al., 2013). Multiple gestations have also been identified as linked to the development of ICP (Lausman et al., 2006; 2008), but there are only a few studies focused on ICP with multiple gestations. One large retrospective cohort study demonstrated increased risks of adverse perinatal outcomes in ICP twin pregnancies (Liu et al., 2015). This study was carried out in a more economically dynamic area in China, and more attention was paid to the fetal outcomes. Sichuan is a populous province in the south-west part of China with a high incidence of ICP. Our study of multiple

RECEIVED 28 April 2016; ACCEPTED 12 July 2016. First published online 28 September 2016.

ADDRESS FOR CORRESPONDENCE: Hu Yayi, Department of Obstetrics and Gynecology, West China Second University Hospital, Sichuan University, Chengdu, Sichuan, China. E-mail: yayi.hu@163.com 
pregnancies of ICP in Sichuan is a reflection of this disease in China, and also serves to fill in the gap of ICP research in low-income regions.

Due to the development and increase in usage of assisted reproductive technology (ART), the incidence of multiple births has risen steadily over the past three decades (International Committee for Monitoring Assisted Reproductive Technology, 2013; Scholten et al., 2015). Recent research found that ART multiple gestation infants were as high as $14.7-29.0 \%$ in different countries (Scholten et al., 2015). The proportion of births from ART in mainland China was about $1 \%$ in 2011 (Yang et al., 2014). Despite the maternal and fetal problems rooted in multiple pregnancies, ART seems to be a single risk factor for substantial excess perinatal morbidities and many other maternal and neonatal adverse events. Complications of multiple pregnancies may be affected or compounded by the presence of ICP; the role of ART in these patients also needs to be explored. Given this background, we sought to ascertain the interaction effect of ICP and ART in twin pregnancies. We aim to investigate the association of ICP with maternal and neonatal complications in ART pregnancies as well as in spontaneous pregnancies (SP). Also, the influence of ART in ICP patients was analyzed.

\section{Materials and Methods}

This retrospective cohort study was undertaken at West China Second University Hospital of Sichuan University in Chengdu, China, following approval from the Ethics Committee. All twin pregnancies delivered above 28 gestational weeks in our hospital from January 2013 to May 2015 were included. International Classification of Diseases, 10 th version (ICD-10) codes were used to identify twin pregnancies complicated by ICP. All the clinical data were obtained from the electronic medical records system.

All cases of ICP were confirmed by demonstration of serum TBA above $10 \mathrm{mmol} / \mathrm{L}$, raised liver transaminase enzymes in association with pruritus, and no additional identifiable cause for their liver dysfunction (Royal College of Obstetricians and Gynaecologists, 2011; Williamson \& Geenes, 2014). Severe ICP was defined according to TBA levels as $>40 \mathrm{mmol} / \mathrm{L}$ (Geenes et al., 2014; Glantz et al., 2004; Raz et al., 2015; Royal College of Obstetricians and Gynaecologists, 2011; Williamson \& Geenes, 2014). Patients were excluded if their pruritus could be attributed to causes other than ICP, or if they had gallstones, cholecystitis, or liver cirrhosis. All patients were screened by lab tests for the infection of hepatitis virus, Epstein Barr virus, and cytomegalovirus. Methods of in vitro fertilization, intracytoplasmic sperm injection, and homologous or heterologous intrauterine insemination conceived twin pregnancies were all included in the ART group.

Data on maternal demographics, obstetric, and medical history were collected for all women, and data on serum biochemistry, management, and monitoring were collected for the ICP cases. Gestational age was calculated with reference to the first trimester ultrasound scan. First trimester ultrasound examination was also used to determine the chorionicity. Maternal complications such as preeclampsia, GDM, and premature rupture of membrane (PROM), placenta previa, placental abruption, postpartum hemorrhage, cesarean delivery rate, and meconium stained amniotic fluid (MSAF) were recorded. The perinatal outcomes studied were intrauterine fetal demise, preterm birth, birth weight, Apgar scores, neonatal intensive care unit (NICU) admission rate, and complications of respiratory tract of the newborns. Preeclampsia was defined according to the American College of Obstetricians and Gynecologists (ACOG) Practice Bulletin 2002 (ACOG, 2002). GDM was diagnosed by the $75 \mathrm{~g}$ oral glucose tolerance test (OGTT) recommended by the American Diabetes Association (2013). Postpartum hemorrhage was defined according to the ACOG practice bulletin 2006 (ACOG, 2006). Fetal growth restriction was defined $<10$ th centile in either baby. A low Apgar score was defined as a score of $<7$ at $5 \mathrm{~min}$.

Data were anonymized and double entered into a customized database. Statistical analysis was performed using SPSS version 19.0 (IBM, Amonki, NY, USA). We compared ICP with non-ICP twin pregnancies. Pregnancies conceived by ART and SP were analyzed separately in subgroup analysis. Comparisons of maternal and fetal outcomes on the difference of severity and onset time of ICP as well as conceiving methods were performed in ICP pregnancies. The effect of ART on maternal and fetal outcomes was also analyzed. A two-tailed $t$ test and a $\chi^{2}$ test were used to compare the clinical characteristics between groups. Independent risk factors were analyzed and identified by univariate and multivariate logistic regression analyses, in which possible confounding factors (maternal age, gravidity, parity, pregestation, body mass index [BMI], chorionicity, and conceiving method) were taken into account. Multiple linear regression was also used in some perinatal outcomes. When analyzing ART effect, we also took socio-economic factors into the multivariate analysis. The unit of analysis was each pregnancy for both the maternal outcomes and the fetal outcomes. As for birth weight, average weight was calculated of the two live newborns; in the case of one deceased fetus, only the weight of the live one was used. $p<$ .05 was considered statistically significant.

\section{Results}

There were a total of 1,543 patients with twin pregnancies delivered in this study: 71 twin gestations were excluded due to gestational age $<28$ weeks at delivery, unconfirmed chorionicity, and loss of data, and 1,472 twin gestations were included in the analysis. ICP was diagnosed in 362 patients and 677 patients conceived using ART (Figure 1). Maternal characteristics were compared between ICP and 
TABLE 1

Baseline Maternal Characteristics

\begin{tabular}{|c|c|c|c|}
\hline Characteristic & Patients with ICP $(n=362)$ & Patients without ICP $(n=1,110)$ & $p$ value \\
\hline Age $(y)$, mean $(S D)$ & $30.80 \pm 4.73$ & $30.23 \pm 4.8$ & .052 \\
\hline Pregestation BMI $\left(\mathrm{kg} / \mathrm{m}^{2}\right)$, mean $(S D)$ & $21.31 \pm 2.85$ & $21.27 \pm 2.83$ & .880 \\
\hline Gravidity, n (\%) & & & .291 \\
\hline 1 & $146(40.3 \%)$ & $439(39.5 \%)$ & \\
\hline 2 & $95(26.2 \%)$ & $285(25.7 \%)$ & \\
\hline$\geq 3$ & $121(33.4 \%)$ & $386(34.8 \%)$ & \\
\hline Parity, $n(\%)$ & & & .073 \\
\hline 1 & $299(82.6 \%)$ & $874(78.7 \%)$ & \\
\hline 2 & $58(16.0 \%)$ & $204(18.4 \%)$ & \\
\hline$\geq 3$ & $5(1.4 \%)$ & $32(2.9 \%)$ & \\
\hline$\overline{\text { Dichorionic, } n(\%)}$ & $295(81.5 \%)$ & $798(71.9 \%)$ & $.001^{a}$ \\
\hline Twins conceived by ART, $n(\%)$ & $191(52.8 \%)$ & $486(43.8 \%)$ & $.003^{\mathrm{a}}$ \\
\hline Insurance, $n(\%)$ & $230(63.5 \%)$ & $690(62.2 \%)$ & .639 \\
\hline \multicolumn{4}{|l|}{ Education, $n(\%)$} \\
\hline College and above & $209(57.7 \%)$ & $657(59.2 \%)$ & .625 \\
\hline \multicolumn{4}{|l|}{ Area of residence, $n(\%)$} \\
\hline Metropolitan & $191(52.8 \%)$ & $602(54.2 \%)$ & .812 \\
\hline Remote & $131(36.2 \%)$ & $397(35.8 \%)$ & \\
\hline Rural & $40(11.0 \%)$ & $111(10.0 \%)$ & \\
\hline
\end{tabular}

Note: $\mathrm{BMI}=$ body mass index; $\mathrm{ART}=$ assisted reproductive techniques; ICP = intrahepatic cholestasis of pregnancy; $\mathrm{SD}=\mathrm{standard}$ deviation. ${ }^{a}$ Denotes significant values if $p<.05$.

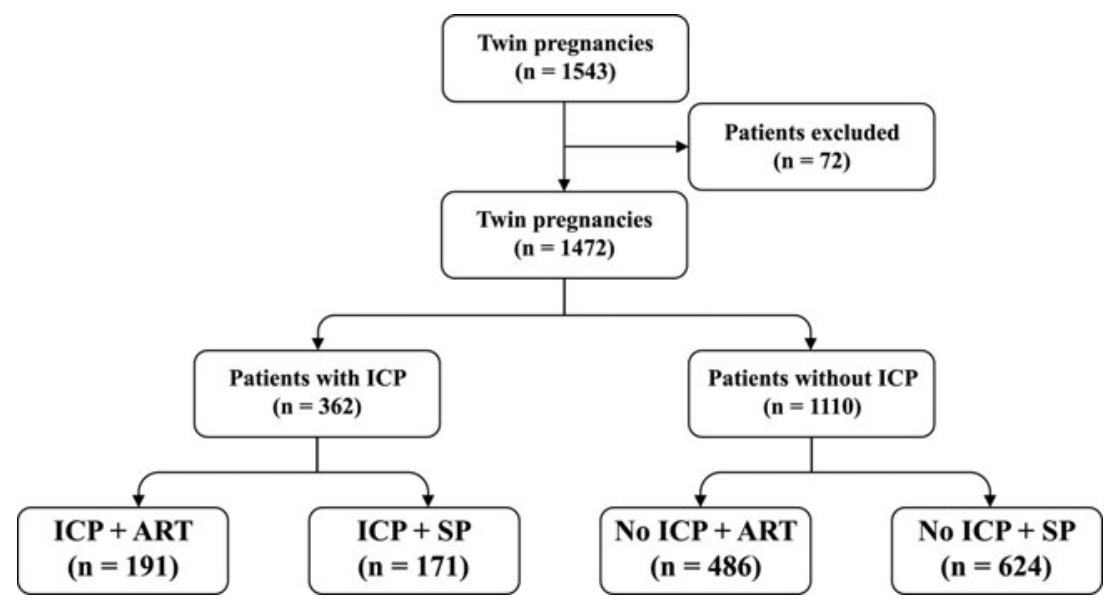

\section{FIGURE 1}

Flow diagram showing the cases ascertainment and groups of included patients.

non-ICP twin sets. Women with ICP were more likely to be conceived by ART (52.8\% vs. $43.8 \%$; $p=.003$ ), and have dichorionic pregnancies $(81.5 \%$ vs. $71.9 \% ; p=.001)$. Maternal age, gravidity, parity, pregestation BMI, and socio-economic factors such as insurance, education, and area of residence did not differ significantly between the two groups (Table 1).

Except for lactate dehydrogenase measured at the time of diagnosis, there were no significant differences of liver enzymes and serum TBA between ART and SP of ICP. Serum TBA, alanine transaminase, and aspartate transaminase were not different at the time of diagnosis or at the time of delivery. The gestational weeks for the diagnosis of ICP (32.2 \pm 3.6 vs. $32.4 \pm 3.7$ weeks in ART and SP, respectively; $p=.75)$ or peak levels of serum TBA (35.9 \pm 34.9 vs. 35.3 $\pm 33.1 \mu \mathrm{mol} / \mathrm{l}$, respectively in ART and SP; $p=.87)$ were not different, either. Ursodeoxycholic acid was given to all the ICP patients, and women with abnormal liver functions were also treated with S-adenosyl-L-methionine.

Regarding maternal complications, there were significantly higher rates of preeclampsia in the ICP population in our unadjusted analysis; the difference remained significant following adjustment for potential confounding factors (14.1\% vs. $7.9 \%$; aOR 1.96; 95\% CI 1.35, 2.85). There was also an increased risk of MSAF (14.4\% vs. 5.6\%; aOR 3.10; 95\% CI 2.10, 4.61). The incidence of PROM was lower in ICP patients (19.3\% vs. $25.1 \%$; aOR 0.71 ; $95 \%$ CI $0.53,0.96$ ). Regarding perinatal outcomes, preterm deliveries $(83.4 \%$ vs. $64.8 \%$; aOR 3.20 ; $95 \%$ CI $2.35,4.37$ ) were more likely in ICP twins. The risk of cesarean delivery was higher in ICP pregnancies in our unadjusted analysis; however, after controlling for the confounding factors, the difference has no 
TABLE 2

Maternal and Fetal Outcomes in ICP and Non-ICP Pregnancies

\begin{tabular}{|c|c|c|c|c|}
\hline Outcomes & $\operatorname{ICP}(n=362)$ & Non-ICP $(n=1110)$ & Unadjusted RR [95\% Cl] & Adjusted $\mathrm{OR}^{\mathrm{a}}[(95 \% \mathrm{Cl}]$ \\
\hline \multicolumn{5}{|l|}{ Maternal outcomes, $n(\%)$} \\
\hline Preeclampsia & $51(14.1 \%)$ & $88(7.9 \%)$ & $1.90[1.31,2.75]^{b}$ & $1.96[1.35,2.85]^{b}$ \\
\hline PROM & $70(19.3 \%)$ & $279(25.1 \%)$ & $0.71[0.53,0.96]^{\mathrm{b}}$ & $0.71[0.53,0.96]^{b}$ \\
\hline Gestational diabetes & $104(28.7 \%)$ & $279(25.1 \%)$ & $1.20[0.92,1.57]$ & $1.13[0.86,1.49]$ \\
\hline Placental abruption & $3(0.8 \%)$ & $25(2.3 \%)$ & $0.36[0.11,1.21]$ & $0.34[0.10,1.15]$ \\
\hline Placental previa & $29(8.0 \%)$ & $83(7.5 \%)$ & $1.08[0.69,1.67]$ & $1.03[0.66,1.61]$ \\
\hline Postpartum hemorrhage & $36(9.94 \%)$ & $91(8.19 \%)$ & $1.24[0.82,1.86]$ & $1.18[0.78,1.78]$ \\
\hline Cesarean delivery & $320(88.4 \%)$ & $926(83.4 \%)$ & $1.51[1.06,2.17]^{\mathrm{b}}$ & $1.40[0.96,2.02]$ \\
\hline MSAF & $52(14.4 \%)$ & $62(5.6 \%)$ & $2.84[1.92,4.19]^{\mathrm{b}}$ & $3.10[2.10,4.61]^{\mathrm{b}}$ \\
\hline \multicolumn{5}{|l|}{ Fetal outcomes, $n[\%)$} \\
\hline Delivery $<37$ weeks & 302 (83.4\%) & $719(64.8 \%)$ & $2.74[2.02,3.71]^{b}$ & $3.20[2.35,4.37]^{b}$ \\
\hline Intrauterine fetal demise & $11(3.0 \%)$ & $58(5.2 \%)$ & $0.57[0.30,1.10]$ & $0.64[0.33,1.25]$ \\
\hline FGR & $37(10.2 \%)$ & $96(8.6 \%)$ & $1.20[0.81,1.79]$ & $1.31[0.87,1.96]$ \\
\hline 5 min Apgar $<7$ & $4(1.1 \%)$ & $21(1.9 \%)$ & $0.58[0.20,1.70]$ & $0.61[0.21,1.80]$ \\
\hline Respiratory complications & $69(19.1 \%)$ & $242(21.8 \%)$ & $0.85[0.63,1.14]$ & $0.92[0.68,1.24]$ \\
\hline NICU admission & $131(36.2 \%)$ & $397(35.8 \%)$ & $1.02[0.80,1.30]$ & $1.12[0.87,1.44]$ \\
\hline
\end{tabular}

Note: ICP = intrahepatic cholestasis of pregnancy; PROM = premature rupture of membranes; MSAF = meconium-stained amniotic fluid; FGR = Fetal growth restriction; $\mathrm{BMI}=$ body mass index; $\mathrm{Cl}=$ confidence interval; $\mathrm{OR}=$ odds ratio; $\mathrm{RR}=$ risk ratio.

${ }^{a}$ Controlled for maternal age, gravidity, parity, pregestation BMI, chorionicity, conceiving method.

${ }^{\mathrm{b}}$ Denotes significance with a $\mathrm{Cl}$ that does not cross 1 .

statistical significance ( $88.4 \%$ vs. $83.4 \%$; aOR $1.40 ; 95 \%$ CI $0.96,2.02)$. No significant differences were found between groups in other maternal outcomes of rate of gestational diabetes, placental abruption, placental previa, or postpartum hemorrhage. Also, there were no significant differences between ICP and non-ICP twins in intrauterine fetal demise, fetal growth restriction, Apgar score at $5 \mathrm{~min}$, NICU admission, or respiratory complications of neonates. To further preclude the influence of other gestational complications, we also performed multivariate analysis, taking preeclampsia as one of the controlling factors. The rate of delivery was still significantly different between ICP and non-ICP twin sets, as was the rate of MSAF and PROM (Table 2).

To better illustrate the differences in ART and SP twin sets, we further performed subgroup analysis. The rates of preeclampsia, MSAF, and preterm labor remained higher in ICP patients conceived by ART; the same differences were found in SP patients, with a slightly larger OR of preeclampsia and preterm delivery. These differences remained after adjustment for confounding factors. ICP and non-ICP population differed in mean newborn birthweight in subgroup analysis of ART twin pregnancies (2243.49 vs. 2327.13 in ICP and non-ICP, respectively; $p=.037$ ). Multivariate linear regression analysis demonstrated ICP was correlated with a lower birthweight after adjustment of the confounding factors (aOR -93.66; 95\% CI -171.92, -15.40). This is probably due to the early gestational weeks at delivery in ICP twin pregnancies (Figure $2 \mathrm{a}$ and $\mathrm{b}$ ).

Different maternal and fetal outcomes of ART pregnancies and SP are shown in Table 3. Gestational diabetes, placental previa, and postpartum hemorrhage were more common in ART pregnancies, while the incidence of fetal respiratory complications and NICU admission were lower. ART pregnancies also had lower rates of preterm deliveries at 34 weeks (Table 3). An analysis limited to pregnancies complicated by ICP did not show a difference in the risk of preeclampsia or MSAF based on conceiving methods. Incidence of gestational diabetes was not significantly different in ART versus SP pregnancies after adjustment for confounding factors ( $33.5 \%$ vs. $23.4 \%$; aOR 1.17 ; $95 \%$ CI 0.66 , 2.06). ART-conceived ICP twin pregnancies have a lower risk of delivery before 37 weeks ( $79.6 \%$ vs. $87.7 \%$; aOR 0.48 ; $95 \%$ CI $0.24,0.94)$, NICU admission of newborns (31.4\% vs. $41.5 \%$; aOR 0.61 ; $95 \%$ CI $0.38,0.99$ ), and neonatal respiratory complications ( $15.2 \%$ vs. $23.4 \%$; aOR 0.53 ; $95 \%$ CI $0.29,0.95)$; the significant difference remained even when adjusting for the gestational weeks at delivery (Figure 2c).

Significant relationships were found between the onset time of ICP and PROM, placenta previa, preterm delivery, NICU admission, and neonatal respiratory complications. We also performed analysis concerning the influence of ICP severity. Compared with the mild and moderate ICP cases, higher risks of MSAF ( $20.6 \%$ vs. $12.1 \%$; aOR 1.92 ; $95 \%$ CI $1.03,3.58)$ and preterm delivery $(91.8 \%$ vs. $80.4 \%$; aOR 2.80 ; $95 \%$ CI $1.26,6.23$ ) were observed in the severe group. Linear logistic regression indicates that the neonatal birthweight was influenced by the onset time and severity of ICP, which is lower in both the early onset ICP cases (aOR -211.83; 95\% CI -294.39, -129.28) and the severe ICP cases (aOR -97.51; 95\% CI -191.78, -3.23) (Table 4).

\section{Discussion}

This study demonstrates the association of ICP and ART in twin pregnancies. Significant correlations between ICP and adverse maternal or neonatal outcomes were found. The present results are in accordance with several published studies that reported a higher risk of preeclampsia, preterm delivery, and MSAF (Brouwers et al., 2015; Geenes 
TABLE 3

Maternal and Fetal Outcomes in ART and SP Pregnancies

\begin{tabular}{|c|c|c|c|c|}
\hline Outcomes & $\operatorname{ART}(n=677)$ & $\mathrm{SP}(n=795)$ & Unadjusted RR [95\% Cl] & Adjusted $\mathrm{OR}^{\mathrm{a}}[95 \% \mathrm{Cl}]$ \\
\hline \multicolumn{5}{|l|}{ Maternal outcomes, n (\%) } \\
\hline Gestational diabetes & $212(31.3 \%)$ & $171(21.5 \%)$ & $1.66[1.32,2.10]^{b}$ & $1.50[1.14,1.96]^{b}$ \\
\hline Placental previa & $65(9.6 \%)$ & $47(5.9 \%)$ & $1.69[1.14,2.50]^{b}$ & $1.80[1.15,2.83]^{b}$ \\
\hline Postpartum hemorrhage & $78(11.5 \%)$ & 49 (6.2\%) & $1.98[1.37,2.88]^{b}$ & $1.99[1.30,3.00]^{b}$ \\
\hline \multicolumn{5}{|l|}{ Fetal outcomes, n (\%) } \\
\hline Delivery $<34$ weeks & $110(16.2 \%)$ & $200(25.2 \%)$ & $0.58[0.45,0.75]^{b}$ & $0.72[0.54,0.97]^{b}$ \\
\hline Delivery $<37$ weeks & $428(63.2 \%)$ & $593(74.6 \%)$ & $0.59[0.47,0.73]^{b}$ & $0.78[0.61,1.02]$ \\
\hline Respiratory complications & $114(16.8 \%)$ & $197(24.8 \%)$ & $0.62[0.48,0.80]^{b}$ & $0.73[0.55,0.98]^{\mathrm{b}}$ \\
\hline NICU admission & $200(29.5 \%)$ & $328(41.3 \%)$ & $0.60[0.48,0.74]^{\mathrm{b}}$ & $0.74[0.58,0.94]^{\mathrm{b}}$ \\
\hline
\end{tabular}

Note: $\mathrm{ART}=$ assisted reproductive techniques; $\mathrm{SP}=$ spontaneous pregnancies; $\mathrm{BMI}=$ body mass index; $\mathrm{Cl}=$ confidence interval; $\mathrm{OR}=$ odds ratio; $\mathrm{RR}=$ risk ratio; $\mathrm{OR}=$ odds ratio.

${ }^{a}$ Controlled for maternal age, gravidity, parity, pregestation BMI, chorionicity, insurance, education, and residence.

${ }^{b}$ Denotes significance with a $\mathrm{Cl}$ that does not cross 1.
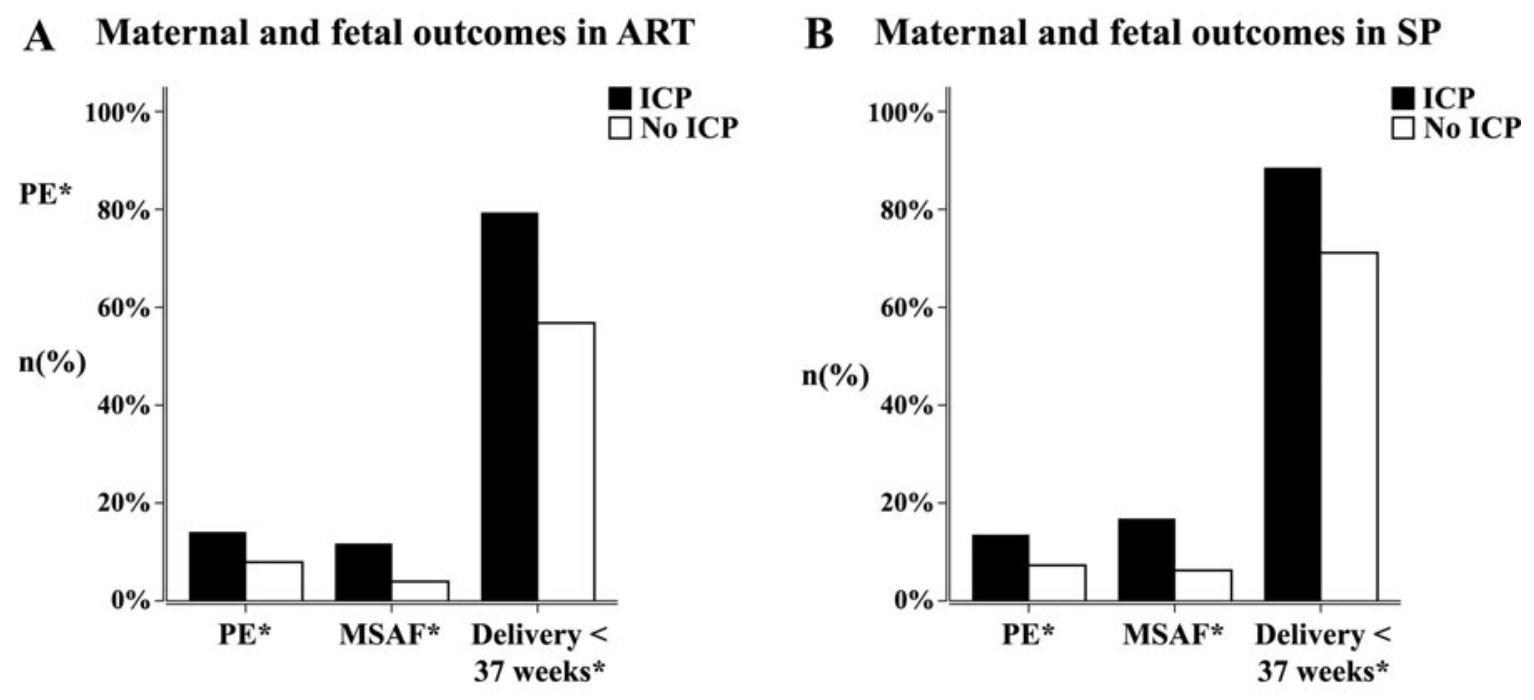

C Fetal outcomes in ICP

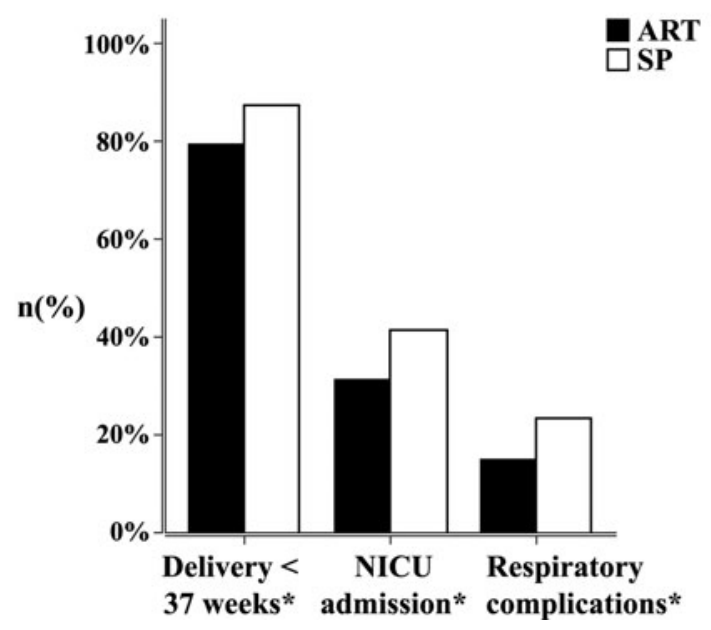

\section{FIGURE 2}

Maternal and fetal outcomes in pregnancies conceived by ART and spontaneous pregnancies (a and b); fetal outcomes in ICP pregnancies (c). (a) Different incidences of preeclampsia (PE), meconium-stained amniotic fluid (MSAF), and preterm delivery in ICP and non-ICP pregnancies conceived by ART; all these differences were statistically significant. (b) Different incidences of preeclampsia (PE), meconium stained amniotic fluid (MSAF), and preterm delivery in ICP and non-ICP spontaneous pregnancies; all these differences were statistically significant. (c) Different incidences of preterm labor, NICU admission, and respiratory complications in ART-conceived pregnancies and spontaneous pregnancies in ICP patients; all these differences were statistically significant. 
TABLE 4

Maternal and Fetal Outcomes in Early and Late Onset ICP Patients

\begin{tabular}{|c|c|c|c|c|}
\hline Outcomes & Early onset $(n=155)$ & Late onset $(n=207)$ & Unadjusted RR [95\% Cl] & Adjusted $^{\mathrm{a}}$ OR $[95 \% \mathrm{Cl}]$ \\
\hline \multicolumn{5}{|l|}{ Maternal outcomes, $n(\%)$} \\
\hline PROM & $43(27.7 \%)$ & $27(13.0 \%)$ & $2.56[1.50,4.37]^{\mathrm{b}}$ & $2.85[1.64,4.98]^{b}$ \\
\hline Placental previa & $18(11.6 \%)$ & $11(5.3 \%)$ & $2.34[1.07,5.11]^{b}$ & $2.44[1.10,5.42]^{b}$ \\
\hline \multicolumn{5}{|l|}{ Fetal outcomes, $n(\%)$} \\
\hline Delivery $<37$ weeks & $142(91.6 \%)$ & $160(77.3 \%)$ & $3.21[1.67,6.17]^{b}$ & $3.56[1.81,6.98]^{b}$ \\
\hline Respiratory complications & $40(25.8 \%)$ & $29(14.0 \%)$ & $2.14[1.25,3.64]^{\mathrm{b}}$ & $2.25[1.30,3.89]^{\mathrm{b}}$ \\
\hline NICU admission & $74(47.7 \%)$ & $57(27.5 \%)$ & $2.40[1.55,3.73]^{b}$ & $2.54[1.62,4.00]^{b}$ \\
\hline
\end{tabular}

Note: $\mathrm{ART}=$ assisted reproductive techniques; $\mathrm{BMI}=$ body mass index; $\mathrm{Cl}=$ confidence interval; $\mathrm{OR}=$ odds ratio; $\mathrm{RR}=$ risk ratio; $\mathrm{OR}=$ odds ratio. ${ }^{a}$ Controlled for maternal age, gravidity, parity, pregestation BMI, chorionicity and conceiving method.

${ }^{b}$ Denotes significance with a confidence interval that does not cross 1 .

et al., 2014; Raz et al., 2015). Although the outcome of twin pregnancies resulting from ART has been the subject of controversy in the literature, our study demonstrated the impact of conceiving method on twin pregnancies. The findings indicate that patients with early-onset ICP or severe ICP had higher possibilities of preterm delivery, MSAF, and lower birth weight, and reveal the requirement of closer monitoring in these patients.

Epidemiologic surveys show significant regional differences of ICP prevalence. It varies from $0.1-1.5 \%$ in Europe to $9.2-15.6 \%$ in South American countries, such as Bolivia or Chile. It is particularly high in native Araucanians in Chile (28\%) (Milkiewicz et al., 2002; Wang et al., 2007; Williamson \& Geenes, 2014). A retrospective study analyzed ICP patients in our hospital from 1991 to 2000 and found the rate of ICP was as high as 5.2\% in singleton pregnancies (Wang et al., 2007). Apart from regional factors, multiple gestations were considered to be another risk factor for ICP because of higher levels of estrogen and progesterone (Gonzalez et al., 1989; Lausman et al., 2006; 2008; Ozkan et al., 2015; Reyes \& Sjovall, 2000).One study reported the incidence of ICP was $20.9 \%$ in twin pregnancies (Gonzalez et al., 1989). The incidence of ICP in our patients was as high as $24.6 \%$. As an important medical center in the south-west part of China, the public acceptance and approval also contributes to this high rate of ICP.

The exaggeration of atherogenic-like response, including insulin resistance and dyslipidemia, is one of the basic adaptive changes in pregnant women, and may manifest itself as preeclampsia or GDM (Kaaja \& Greer, 2005). Some common pathogenetic pathways seem to be shared by preeclampsia and GDM (Kaaja \& Greer, 2005; Martineau et al., 2014; 2015), especially in women with higher BMI whose metabolic pathways are disturbed, which can cause adverse maternal or fetal outcomes (Cnattingius et al., 1998; Stothard et al., 2009). In our multivariate analysis, pregestation $\mathrm{BMI}$ is the common contributor to both preeclampsia (aOR 1.10) and GDM (aOR 1.12). The similar finding of obesity as a risk factor for preeclampsia or GDM was inconsistent with other studies (Al-Obaidly et al., 2014; Scifres et al., 2015; Somprasit et al., 2015). A retrospective study in Canada included 504 twin pregnancies. When participants were divided into four groups according to pregestation $\mathrm{BMI}$, researchers found an increased risk of $\mathrm{PE}$ and GDM in the obese patients group (Al-Obaidly et al., 2014).

Except for pregestation BMI, the elevated TBA levels may act synergistically to cause preeclampsia or impaired glucose tolerance (Martineau et al., 2014; 2015; Ozkan et al., 2015; Williamson \& Geenes, 2014). On the one hand, elevated serum bile acids may cause malfunction of endothelial cells and renal disturbances (Perez \& Briz, 2009; Smolarczyk et al., 2000). It can also trigger increased oxidative stress reaction, and leads to the up-regulation of soluble tyrosine kinasee1, which is considered to be a major contributor to the pathogenesis of preeclampsia (Anderson et al., 2012; De Vivo et al., 2008; Hu et al., 2015; Li et al., 2005; Maynard et al., 2003). The increased incidence of preeclampsia in ICP patients was proven in singleton pregnancies (Geenes et al., 2014; Goulis et al., 2004; Lee et al., 2006; Marschall et al., 2012; Wikstrom et al., 2013), but studies reported in the literature are not consistent on the incidence of preeclampsia in ICP twin pregnancies. Lausman et al. (2008) found no difference in the incidence of preeclampsia in ICP pregnancies in 263 multiple pregnancies in Canada (Lausman et al., 2008). Another study of 124 twin pregnancies revealed that the incidence of preeclampsia were almost five-fold in ICP patients compared to the controls (Raz et al., 2015). Moreover, Raz et al. (2015) found preeclampsia was concomitant with ICP severity at initial diagnosis, and occurred most often 2-4 weeks after the diagnosis of ICP. In our study of 1,543 twin pregnancies, the correlation of diagnosis time of ICP and preeclampsia was not found, but incidence of preeclampsia was significantly higher (almost two-fold) in ICP twin sets.

On the other hand, bile acids were reported to activate the farnesoid X (FXR) receptor and promote insulin release (Ma et al., 2006). Disruption in homeostatic pathways for glucose balance was observed in ICP pregnancies (Martineau et al., 2014; 2015). Studies targeting singletons indicated an elevated risk of GDM in ICP pregnancies with an OR of 1.6-2.8 (Diken et al., 2014; Martineau et al., 2015). However, the incidence of GDM was not different in women predisposed to ICP in our study. Multivariate 
analysis revealed the major contributors to GDM were pregestation BMI as well as the patient's age. This may indicate the possibility of the inability in glucose homeostasis regulation with advancing age, which may contribute more to the pathogenesis of GDM, in addition to the known effects upon bile acid metabolism. Interventions to control weight gain should be used to improve outcomes in these high-risk women.

Except for maternal complications, the accumulating data indicated higher risk of fetal complications relating to elevated circulating bile acids (Geenes et al., 2014; Lausman et al., 2008; Puljic et al., 2015; Shemer et al., 2013; Wikstrom et al., 2013). In our study, of the 362 ICP twin pregnancies after 28 weeks of gestation were included, we found that ICP was correlated with higher risks of preterm labor and MSAF, while early onset ICP had higher incidence of PROM. Other studies with smaller numbers of participants with twin pregnancies had different findings (Gonzalez et al., 1989; Lausman et al., 2008). In ICP pregnancies, the transplacental gradient for excretion of bile acids from the fetus to mother is reversed, leading to the accumulation of the toxic compounds in the fetal serum as well as amniotic fluid (Brites, 2002; Reyes \& Sjovall, 2000; Williamson $\&$ Geenes, 2014). A dose-dependent bile acid effect on myometrial contractility was demonstrated in rodents (Campos et al., 1988). Bile acids can cause increased expression and response of the oxytocin receptor in human myometrial cells (Germain et al., 2003; Israel et al., 1986). Preterm labor may be explained by increased uterine contractility in the third trimester of ICP patients (Zhao et al., 2014). Alterations of hepatic metabolites in amniotic fluid in ICP pregnancies were observed (Menon et al., 2014). Bile salts could react with surface-active phospholipid and reduce the hydrophobicity and lubricity of amniotic and chorionic epithelium (Hills, 1994). We found women with early-onset ICP had a higher risk of PROM compared with the lateonset group in the subanalysis focusing on ICP pregnancies. However, when compared with the non-ICP pregnancies, the incidence of PROM was not higher, but this correlation was not found when we took in gestational weeks at delivery in multivariate analysis. Over-activated gut motility stimulated by bile acids was found in animal studies (Campos et al., 1986), which may explain MSAF.

Although our study did not find the hypodevelopment of fetal lungs, other studies revealed the altered composition and structure of fetal pulmonary surfactant in ICP pregnancies, and the changes of fetal pulmonary surfactant were correlated with the bile acids in amniotic fluid as well as in fetal cord blood (Yu et al., 2011; Zhang et al., 2015). Intratracheal injection of bile acids in rabbits can cause atelectasis, eosinophilic infiltration, and the formation of hyaline membrane in lung (Kaneko et al., 1990). Hypodevelopment of fetal lungs is the most common reason for NICU admission in ICP pregnancies, however, the most disastrous and unacceptable consequence of ICP pregnancies is stillbirth.
The rate of stillbirth in women with ICP is reported to be 1.5-7.0\% (Geenes et al., 2014; Jin et al., 2015; Kawakita et al., 2015; Rioseco et al., 1994); especially in patients with a TBA level above $100 \mathrm{mmol} / \mathrm{L}$, the rate of stillbirth was as high as 10-15\% (Brouwers et al., 2015; Kawakita et al., 2015). In our study, the stillbirth rate was $3.0 \%$ in ICP twin pregnancies. Postmortem studies of stillborn neonates provided evidence of acute anoxia (Reid et al., 1976), which may be explained by sudden arrhythmias in neonates or marked vasoconstriction of placental chorionic vessels caused by bile acids (Al Inizi et al., 2006; Miragoli et al., 2011; Sepulveda et al., 1991). Different myocardial tissue velocities of both mitral and tricuspid valves in ICP fetuses were also found using tissue Doppler imaging (Ataalla et al., 2016).

Like Raz et al. (2015), we took 32 gestational weeks as the cut-off time point to determine the onset time of ICP. In our linear regression analysis, we did not find the correlation of ICP and birth weight, which is similar in previous reported results in singletons. However, after performing a subanalysis in ICP pregnancies, concentrating on severity and onset time, the differences were revealed. Patients from the severe ICP group had higher frequencies of preterm deliveries and MSAF than the mild group. The same results were found in the early-onset group compared with late-onset group. These findings indicate that the increased TBA correlates with poorer fetal outcomes, and the correlation is reinforced by time. In the present study, we also revealed a higher incidence of newborns in the early-onset group experienced respiratory problems and NICU admission. These findings indicate the possibility that it is the time of the disease, rather than the level of circulating TBA, that might be more disastrous to the fetal lungs.

The different incidences of ICP in ART pregnancies were rarely reported. In Finland, researchers included 225 IVF pregnancies (including multiple pregnancies) and 671 control pregnancies. The risk ratio of ICP for IVF singletons pregnancies was 3.8 (95\% CI 1.0, 15.0; Koivurova et al., 2002). The authors attributed this phenomenon to metabolic disturbances regarding infertility itself. Zamah et al. (2008) reported two patients with first-trimester cholestasis of pregnancy after IVF. The elevated serum bile acids level was associated with markedly elevated maternal serum estrogen levels caused by ovarian hyperstimulation syndrome (Zamah et al., 2008). The abnormal liver function and higher incidence of ICP in ART pregnancies were also found in Kopylov's (2013) study. They concluded that a potential pathogenetic mechanism for this correlation was rooted in the hormonal hyperstimulation syndrome occurring with ART treatments (Kopylov et al., 2013). In our study, the rate of ICP was higher in ART pregnancies. But in the separate analysis of ART and SP pregnancies, we did not find much difference in the influence of ICP between these two groups of patients. There were no huge differences of the aOR for preeclampsia, MSAF, and preterm delivery between ART and SP pregnancies. 
Several studies comparing the neonatal and maternal outcome of ART and SP twin pregnancies have reported conflicting results (Bamberg et al., 2012; Fan et al., 2013; Moini et al., 2012; Qin et al., 2015; Stern et al., 2015; Szymusik et al., 2012). Some studies reported that complications, such as GDM and preterm delivery were significantly more common in ART twins (Bamberg et al., 2012; Moini et al., 2012; Qin et al., 2015; Stern et al., 2015). Similar to these studies, our research revealed higher risks for GDM, placental previa, and postpartum hemorrhage for ART pregnancies. But, as for fetal outcomes, the incidence of preterm deliveries, fetal respiratory complications, and NICU admission were lower in ART pregnancies, even when taking socio-economic factors into consideration. The same results were found in subgroup analysis of ICP pregnancies. Although many studies indicated a higher preterm delivery rate in ART pregnancies, there were still some studies with dissenting results. A Polish study found similar rates of preterm deliveries of ART and SP pregnancies (Szymusik et al., 2012). Fan et al. (2013) included 162 ART dichorionic twin pregnancies together with 213 SP in Hubei province, China, and revealed a slightly lower incidence of preterm deliveries in the ART group. Our finding of lower rates in some adverse fetal outcomes might be explained by the larger gestational weeks at delivery in ART patients, which could lead to better development of fetal lungs (Gerber, 2015; Huddle et al., 2016). The difference in the rate of monochorionic twin pregnancies also contributes to the different outcomes. Many studies have shown that dichorionic twin pregnancies have a better prognosis and lower rates of preterm deliveries than monochorionic twin pregnancies (Burgess et al., 2014; Carter et al., 2015; D’Arpe et al., 2016; Masheer et al., 2015). For ART pregnancies, the rate of monochorionic pregnancy was only $6.35 \%$, while the rate of monochorionic pregnancy in spontaneous twin pregnancies was $42.26 \%$ in our study. Better compliance with doctors' suggestions and more attention and care from family members might also lead to better outcomes for ART pregnant women. The long-term outcomes of ART twins are still largely unknown. Prospective studies are needed to explore the influence of ART.

This study addresses a significant gap in the literature on the outcomes of ICP twin pregnancies in Southwest China, where the incidence of ICP is higher compared with other places. The few existing studies on ICP twin pregnancies have been confined to high-income countries. To the best of our knowledge, the number of ICP twin pregnancies included in our study is the largest. Patients deriving from a single medical center ascertained the same diagnostic criteria, treatment, and prenatal monitoring. This study has limitations that should be noted. Medical records rather than direct patient interview were used to obtain the data. This resulted in lack of data on several maternal variables, such as educational level, family income, and smoking status. As a retrospective study in a large tertiary hospital, our results cannot be generalized to patients in community settings or to those delivering at home, who are the most common cases in low-income places in China.

In conclusion, our findings of a strong association of ICP and adverse maternal and fetal outcomes in twin pregnancies support the current practice of close antenatal monitoring of ICP twin pregnancies. More focus should also be paid on severe ICP and early-onset ICP to improve the prediction and treatment of these patients.

\section{Acknowledgments}

This research was funded by the National Natural Science Foundation of China (Number: 81200452) and supported by the Science and Technology Department of Sichuan Province (Number: 2015SZ0139). The authors declare no conflict of interest.

\section{References}

Al-Inizi, S., Gupta, R., \& Gale, A. (2006). Fetal tachyarrhythmia with atrial flutter in obstetric cholestasis. International Journal of Gynecology and Obstetrics, 93, 53-54.

Al-Obaidly, S., Parrish, J., Murphy, K. E., \& Maxwell, C. (2014). Maternal pre-gravid body mass index and obstetric outcomes in twin gestations. Journal of Perinatology, 34, 425-428.

American College of Obstetricians and Gynecologists (ACOG). (2002). ACOG practice bulletin. Diagnosis and management of preeclampsia and eclampsia. Number 33, January 2002. Obstetrics and Gynecology, 99, 159-167.

American College of Obstetricians and Gynecologists (ACOG). (2006). ACOG practice bulletin. Clinical management guidelines for obstetrician-gynecologists number 76, October 2006: Postpartum hemorrhage. Obstetrics and Gynecology, 108, 1039-1047.

American Diabetes Association (ADA). (2013). Standards of medical care in diabetes-2013. Diabetes Care, 36, S11-S66.

Anderson, U. D., Olsson, M. G., Kristensen, K. H., Akerstrom, B., \& Hansson, S. R. (2012). Review: Biochemical markers to predict preeclampsia. Placenta, 33, S42-S47.

Ataalla, W. M., Ziada, D. H., Gaber, R., Ossman, A., Bayomy, S., \& Elemary, B. R. (2016). The impact of total bile acid levels on fetal cardiac function in intrahepatic cholestasis of pregnancy using fetal echocardiography: A tissue Doppler imaging study. Journal of Maternal-Fetal and Neonatal Medicine, 29, 1445-1450.

Bamberg, C., Fotopoulou, C., Neissner, P., Slowinski, T., Dudenhausen, J. W., Proquitte, H., ... Henrich, W. (2012). Maternal characteristics and twin gestation outcomes over 10 years: Impact of conception methods. Fertility and Sterility, 98, 95-101.

Brites, D. (2002). Intrahepatic cholestasis of pregnancy: Changes in maternal-fetal bile acid balance and improvement by ursodeoxycholic acid. Annals of Hepatology, 1, 2028. 
Brouwers, L., Koster, M. P., Page-Christiaens, G. C., Kemperman, H., Boon, J., Evers, I. M., ... Oudijk, M. A. (2015). Intrahepatic cholestasis of pregnancy: Maternal and fetal outcomes associated with elevated bile acid levels. American Journal of Obstetrics \& Gynaecology, 212, 100.e101-100.e107.

Burgess, J. L., Unal, E. R., Nietert, P. J., \& Newman, R. B. (2014). Risk of late-preterm stillbirth and neonatal morbidity for monochorionic and dichorionic twins. American Journal of Obstetrics \& Gynaecology, 210, 578.e1-578.e9.

Campos, G. A., Castillo, R. J., \& Toro, F. G. (1988). Effect of bile acids on the myometral contractility of the isolated pregnant uterus. Revista Chilena de Obstetricia y Ginecologia, 53, 229-233.

Campos, G. A., Guerra, F. A., \& Israel, E. J. (1986). Effects of cholic acid infusion in fetal lambs. Acta Obstetricia et Gynecologica Scandinavica, 65, 23-26.

Carter, E. B., Bishop, K. C., Goetzinger, K. R., Tuuli, M. G., \& Cahill, A. G. (2015). The impact of chorionicity on maternal pregnancy outcomes. American Journal of Obstetrics \& Gynaecology, 213, 390.e1-390.e7.

Cnattingius, S., Bergstrom, R., Lipworth, L., \& Kramer, M. S. (1998). Prepregnancy weight and the risk of adverse pregnancy outcomes. New England Journal of Medicine, 338, 147-152.

D’Arpe, S., Franceschetti, S., De Stefano, M. G., D’Amelio, R., Maragno, A. M., Candelieri, M., ... Benedetti Panici, P. (2016). The impact of chorionicity and type of conception on maternal-neonatal outcome in twin pregnancies. Clinical and Experimental Obstetrics \& Gynecology, 43, 88-92.

De Vivo, A., Baviera, G., Giordano, D., Todarello, G., Corrado, F., \& D'Anna, R. (2008). Endoglin, PlGF and sFlt-1 as markers for predicting pre-eclampsia. Acta Obstetricia et Gynecologica Scandinavica, 87, 837-842.

Diken, Z., Usta, I. M., \& Nassar, A. H. (2014). A clinical approach to intrahepatic cholestasis of pregnancy. American Journal of Perinatology, 31, 1-8.

Fan, C., Sun, Y., Yang, J., Ye, J., \& Wang, S. (2013). Maternal and neonatal outcomes in dichorionic twin pregnancies following IVF treatment: A hospital-based comparative study. International Journal of Clinical and Experimental Pathology, 6, 2199-2207.

Geenes, V., Chappell, L. C., Seed, P. T., Steer, P. J., Knight, M., \& Williamson, C. (2014). Association of severe intrahepatic cholestasis of pregnancy with adverse pregnancy outcomes: A prospective population-based case-control study. Нераtology, 59, 1482-1491.

Gerber, A. N. (2015). Glucocorticoids and the lung. Advances in Experimental Medicine and Biology, 872, 279-298.

Germain, A. M., Kato, S., Carvajal, J. A., Valenzuela, G. J., Valdes, G. L., \& Glasinovic, J. C. (2003). Bile acids increase response and expression of human myometrial oxytocin receptor. American Journal of Obstetrics and Gynaecology, 189, 577-582.

Glantz, A., Marschall, H. U., \& Mattsson, L. A. (2004). Intrahepatic cholestasis of pregnancy: Relationships between bile acid levels and fetal complication rates. Hepatology, 40, 467-474.
Gonzalez, M. C., Reyes, H., Arrese, M., Figueroa, D., Lorca, B., Andresen, M., ... Arce, S. (1989). Intrahepatic cholestasis of pregnancy in twin pregnancies. Journal of Hepatology, 9, 84-90.

Goulis, D. G., Walker, I. A. L., de Swiet, M., Redman, C. W. G., \& Williamson, C. (2004). Preeclampsia with abnormal liver function tests is associated with cholestasis in a subgroup of cases. Hypertension In Pregnancy, 23, 19-27.

Hills, B. A. (1994). Further studies of the role of surfactant in premature rupture of the membranes. American Journal of Obstetrics and Gynaecology, 170, 195-201.

Hu, Y. Y., Liu, J. C., \& Xing, A. Y. (2015). Oxidative stress markers in intrahepatic cholestasis of pregnancy: A prospective controlled study. European Review for Medical and Pharmacological Sciences, 19, 3181-3186.

Huddle, M. G., Tekes, A., \& Tunkel, D. E. (2016). Respiratory distress in a neonate. JAMA Otolaryngology-Head \& Neck Surgery, 142, 191-192.

International Committee for Monitoring Assisted Reproductive Technology (ICMART). (2013, July). Preliminary global $A R T$ data for 2009. Paper presented at the European Society of Human Reproduction and Embryology (ESHRE) Annual Scientific Meeting, London.

Israel, E. J., Guzman, M. L., \& Campos, G. A. (1986). Maximal response to oxytocin of the isolated myometrium from pregnant patients with intrahepatic cholestasis. Acta Obstetricia et Gynecologica Scandinavica, 65, 581-582.

Jin, J., Pan, S.-L., Huang, L.-P., Yu, Y.-H., Zhong, M., \& Zhang, G.-W. (2015). Risk factors for adverse fetal outcomes among women with early- versus late-onset intrahepatic cholestasis of pregnancy. International Journal of Gynaecology and Obstetrics, 128, 236-240.

Kaaja, R. J., \& Greer, I. A. (2005). Manifestations of chronic disease during pregnancy. JAMA, 294, 2751-2757.

Kaneko, T., Sato, T., Katsuya, H., \& Miyauchi, Y. (1990). Surfactant therapy for pulmonary edema due to intratracheally injected bile acid. Critical Care Medicine, 18, 77-83.

Kawakita, T., Parikh, L. I., Ramsey, P. S., Huang, C. C., Zeymo, A., Fernandez, M., ... Iqbal, S. N. (2015). Predictors of adverse neonatal outcomes in intrahepatic cholestasis of pregnancy. Journal of Obstetrics and Gynaecology, 213, 570.e571-570.e578.

Koivurova, S., Hartikainen, A. L., Karinen, L., Gissler, M., Hemminki, E., Martikainen, H., ... Jarvelin, M. R. (2002). The course of pregnancy and delivery and the use of maternal healthcare services after standard IVF in Northern Finland 1990-1995. Human Reproduction, 17, 28972903.

Kopylov, U., Avidan, B., Papageorgiou, N. P., Katz, L. H., Sivan, E., Zimlichman, E., ... Maor, Y. (2013). Idiopathic liver function test abnormality in pregnancy is associated with assisted reproduction techniques. Fertility and Sterility, 99, 377-381.

Lausman, A. Y., Al-Yaseen, E., Sam, D., Nitsch, R., Barrett, J. F. R., \& Chan, W.-S. (2008). Intrahepatic cholestasis of pregnancy in women with a multiple pregnancy: An analysis of risks and pregnancy outcomes. Journal of Obstetrics and Gynaecology Canada, 30, 1008-1013. 
Lausman, A., Al-Yaseen, E., Sam, D., Nitsch, R., \& Chan, W.-S. (2006). Intrahepatic cholestasis of pregnancy in multiples: An analysis of risks and pregnancy outcomes. Hypertension In Pregnancy, 25, 26-26.

Lee, R. H., Goodwin, T. M., Greenspoon, J., \& Incerpi, M. (2006). The prevalence of intrahepatic cholestasis of pregnancy in a primarily Latina Los Angeles population. Journal of Perinatology, 26, 527-532.

Li, H., Gu, B., Zhang, Y., Lewis, D. F., \& Wang, Y. (2005). Hypoxia-induced increase in soluble Flt-1 production correlates with enhanced oxidative stress in trophoblast cells from the human placenta. Placenta, 26, 210-217.

Li, L., Zhao, X. Y., Ou, X. J., \& Jia, J. D. (2013). Clinical analysis of introhepatic cholestasis of pregnancy. Chinese Journal of Hepatology, 21, 295-298

Liu, X., Landon, M. B., Chen, Y., \& Cheng, W. (2015). Perinatal outcomes with intrahepatic cholestasis of pregnancy in twin pregnancies. Journal of Maternal-Fetal \& Neonatal Medicine, 1-6.

Ma, K., Saha, P. K., Chan, L., \& Moore, D. D. (2006). Farnesoid $\mathrm{X}$ receptor is essential for normal glucose homeostasis. Journal of Clinical Investigation, 116, 1102-1109.

Marschall, H.-U., Shemer, E. W., Ludvigsson, J. F., \& Stephansson, O. (2012). Intrahepatic cholestasis of pregnancy is not associated with intrauterine fetal death but with gestational diabetes and preeclampsia. Gastroenterology, 142, S912-S913.

Martineau, M. G., Raker, C., Dixon, P. H., Chambers, J., Machirori, M., King, N. M., ... Williamson, C. (2015). The metabolic profile of intrahepatic cholestasis of pregnancy is associated with impaired glucose tolerance, dyslipidemia, and increased fetal growth. Diabetes Care, 38, 243-248.

Martineau, M., Raker, C., Powrie, R., \& Williamson, C. (2014). Intrahepatic cholestasis of pregnancy is associated with an increased risk of gestational diabetes. European Journal of Obstetrics \& Gynecology and Reproductive Biology, 176, 8085.

Masheer, S., Maheen, H., \& Munim, S. (2015). Perinatal outcome of twin pregnancies according to chorionicity: An observational study from tertiary care hospital. Journal of Maternal-Fetal \& Neonatal Medicine, 28, 23-25.

Maynard, S. E., Min, J. Y., Merchan, J., Lim, K. H., Li, J., Mondal, S., ... Karumanchi, S. A. (2003). Excess placental soluble fms-like tyrosine kinase 1 (sFlt1) may contribute to endothelial dysfunction, hypertension, and proteinuria in preeclampsia. Journal of Clinical Investigation, 111, 649658.

Menon, R., Jones, J., Gunst, P. R., Kacerovsky, M., Fortunato, S. J., Saade, G. R., \& Basraon, S. (2014). Amniotic fluid metabolomic analysis in spontaneous preterm birth. Reproductive Sciences, 21, 791-803.

Milkiewicz, P., Elias, E., Williamson, C., \& Weaver, J. (2002). Obstetric cholestasis. BMJ, 324, 123-124.

Miragoli, M., Kadir, S. H., Sheppard, M. N., Salvarani, N., Virta, M., Wells, S., ... Gorelik, J. (2011). A protective antiarrhythmic role of ursodeoxycholic acid in an in vitro rat model of the cholestatic fetal heart. Hepatology, 54, 12821292.
Moini, A., Shiva, M., Arabipoor, A., Hosseini, R., Chehrazi, M., \& Sadeghi, M. (2012). Obstetric and neonatal outcomes of twin pregnancies conceived by assisted reproductive technology compared with twin pregnancies conceived spontaneously: A prospective follow-up study. European Journal of Obstetrics \& Gynecology and Reproductive Biology, 165, 29-32.

Obiechina, N., Okolie, V., Eleje, G., Okechukwu, Z., \& Anemeje, O. (2011). Twin versus singleton pregnancies: The incidence, pregnancy complications, and obstetric outcomes in a Nigerian tertiary hospital. International Journal of Womens Health, 3, 227-230.

Ozkan, S., Ceylan, Y., Ozkan, O. V., \& Yildirim, S. (2015). Review of a challenging clinical issue: Intrahepatic cholestasis of pregnancy. World Journal of Gastroenterology, 21, 71347141.

Perez, M. J., \& Briz, O. (2009). Bile-acid-induced cell injury and protection. World Journal of Gastroenterology, 15, 1677-1689.

Puljic, A., Kim, E., Page, J., Esakoff, T., Shaffer, B., LaCoursiere, D. Y., \& Caughey, A. B. (2015). The risk of infant and fetal death by each additional week of expectant management in intrahepatic cholestasis of pregnancy by gestational age. American Journal of Obstetrics and Gynaecology, 212, 667.e661-667.e665.

Qin, J., Wang, H., Sheng, X., Liang, D., Tan, H., \& Xia, J. (2015). Pregnancy-related complications and adverse pregnancy outcomes in multiple pregnancies resulting from assisted reproductive technology: A metaanalysis of cohort studies. Fertility and Sterility, 103, 14921508.

Rao, A., Sairam, S., \& Shehata, H. (2004). Obstetric complications of twin pregnancies. Best Practice \& Research Clinical Obstetrics \& Gynaecology, 18, 557-576.

Raz, Y., Lavie, A., Vered, Y., Goldiner, I., Skornick-Rapaport, A., Asher, Y. L., ... Rimon, E. (2015). Severe intrahepatic cholestasis of pregnancy is a risk factor for preeclampsia in singleton and twin pregnancies. American Journal of $\mathrm{Ob}$ stetrics and Gynaecology, 213, 395.e1-395e.8.

Reid, R., Ivey, K. J., Rencoret, R. H., \& Storey, B. (1976). Fetal complications of obstetric cholestasis. British Medical Journal, 1(6014), 870-872.

Reyes, H., \& Sjovall, J. (2000). Bile acids and progesterone metabolites in intrahepatic cholestasis of pregnancy. Annals of Medicine, 32, 94-106.

Rioseco, A. J., Ivankovic, M. B., Manzur, A., Hamed, F., Kato, S. R., Parer, J. T., \& Germain, A. M. (1994). Intrahepatic cholestasis of pregnancy: A retrospective case-control study of perinatal outcome. American Journal of Obstetrics and Gynaecology, 170, 890-895.

Royal College of Obstetricians and Gynaecologists. (2011). Obstetric cholestasis (Green-top Guideline No. 43). Retrieved from https://www.rcog.org.uk/en/guidelinesresearch-services/guidelines/gtg43/_RCOG_Models_ Pages_GuidelinesDetailsType.

Scholten, I., Chambers, G. M., van Loendersloot, L., van der Veen, F., Repping, S., Gianotten, J., ... Mol, B. W. J. (2015). Impact of assisted reproductive technology on the 
incidence of multiple-gestation infants: A population perspective. Fertility And Sterility, 103, 179-183.

Scifres, C., Feghali, M., Althouse, A. D., Caritis, S., \& Catov, J. (2015). Adverse outcomes and potential targets for intervention in gestational diabetes and obesity. Obstetrics and Gynecology, 126, 316-325.

Sepulveda, W. H., Gonzalez, C., Cruz, M. A., \& Rudolph, M. I. (1991). Vasoconstrictive effect of bile acids on isolated human placental chorionic veins. European Journal of $\mathrm{Ob}$ stetrics \& Gynecology and Reproductive Biology, 42, 211215.

Shemer, E. A. W., Thorsell, M., Marschall, H.-U., \& Kaijser, M. (2013). Risks of emergency cesarean section and fetal asphyxia after induction of labor in intrahepatic cholestasis of pregnancy: A hospital-based retrospective cohort study. Sexual \& Reproductive Healthcare, 4, 17-22.

Smolarczyk, R., Wojcicka-Jagodzinska, J., Piekarski, P., Romejko, E., \& Czajkowski, K. (2000). The biochemical functions of the renal tubules and glomeruli in the course of intrahepatic cholestasis in pregnancy. European Journal of Obstetrics \& Gynecology and Reproductive Biology, 89, 35-39.

Somprasit, C., Tanprasertkul, C., Rattanasiri, T., Saksiriwutth, P., Wongkum, J., Kovavisarach, E., ... Wuthiwong, J. (2015). High pre-pregnancy body mass index and the risk of poor obstetrics outcomes among Asian women using BMI criteria for Asians by world health organization western pacific region (WPRO): A large cohort study. Journal of the Medical Association of Thailand, 98, S101S107.

Stern, J. E., Luke, B., Tobias, M., Gopal, D., Hornstein, M. D., \& Diop, H. (2015). Adverse pregnancy and birth outcomes associated with underlying diagnosis with and without assisted reproductive technology treatment. Fertility and Sterility, 103, 1438-1445.

Stothard, K. J., Tennant, P. W. G., Bell, R., \& Rankin, J. (2009). Maternal overweight and obesity and the risk of congenital anomalies a systematic review and meta-analysis. JAMA, 301, 636-650.
Szymusik, I., Kosinska-Kaczynska, K., Bomba-Opon, D., \& Wielgos, M. (2012). IVF versus spontaneous twin pregnancies-Which are at higher risk of complications?. Journal of Maternal-Fetal \& Neonatal Medicine, 25, 27252728.

Vogel, J. P., Torloni, M. R., Seuc, A., Betrán, A. P., Widmer, M., Souza, J. P., \& Merialdi, M. (2013). Maternal and perinatal outcomes of twin pregnancy in 23 low- and middle-income countries. PLoS One, 8, e70549.

Wang, X. D., Yao, Q., Peng, B., Zhang, L., Ai, Y., Ying, A. Y., ... Liu, S. Y. (2007). A clinical analysis of intrahepatic cholestasis of pregnancy in 1241 cases. Zhonghua Gan Zang Bing Za Zhi, 15, 291-293.

Wikstrom Shemer, E., Marschall, H. U., Ludvigsson, J. F., \& Stephansson, O. (2013). Intrahepatic cholestasis of pregnancy and associated adverse pregnancy and fetal outcomes: A 12-year population-based cohort study. BJOG, 120, 717-723.

Williamson, C., \& Geenes, V. (2014). Intrahepatic cholestasis of pregnancy. Obstetrics and Gynecology, 124, 120-133.

Yang, X., Li, Y., Li, C., \& Zhang, W. (2014). Current overview of pregnancy complications and live-birth outcome of assisted reproductive technology in mainland China. Fertility and Sterility, 101, 385-391.

Yu, L., Ding, Y. L., \& Wang, C. X. (2011). Relationship between total bile acid concentration and fetal pulmonary surfactant in intrahepatic cholestasis of pregnancy. Zhonghua Fu Chan Ke Za Zhi, 46, 324-328.

Zamah, A. M., El-Sayed, Y. Y., \& Milki, A. A. (2008). Two cases of cholestasis in the first trimester of pregnancy after ovarian hyperstimulation. Fertility and Sterility, 90, 1202.e12071202.e1210.

Zhang, Y., Li, F., Wang, Y., Pitre, A., Fang, Z. Z., Frank, M. W., ... Schuetz, J. D. (2015). Maternal bile acid transporter deficiency promotes neonatal demise. Nature Communications, 6, 8186.

Zhao, P., Zhang, K., Yao, Q., \& Yang, X. (2014). Uterine contractility in intrahepatic cholestasis of pregnancy. Journal Of Obstetrics And Gynaecology, 34, 221-224. 